\title{
Youtubers infantiles: los menores como recurso para generar nuevas tendencias publicitarias
}

\author{
Inmaculada Sánchez-Labella Martín \\ isanchez4@us.es
}

Departamento de Comunicación Audiovisual y Publicidad, Universidad de Sevilla.

Fecha de finalización del trabajo: 18 de marzo de 2020 Recibido: 18 de marzo de 2020 Aceptado: 20 de mayo de 2020.

DOI: https://doi.org/10.26422/aucom.2020.0902.san

\section{Resumen}

Al considerar los trabajos previos publicados por otros autores, el objetivo en este caso es doble. Por un lado, se pretende analizar las marcas que están presentes en los canales de los youtubers infantiles más reconocidos internacionalmente; por otro lado, y dado que ninguna de las investigaciones previas se detiene a estudiar a este grupo como menores que, por su reconocimiento, han terminado convirtiéndose en personajes, este trabajo plantea la necesidad de analizarlos desde el punto de vista de su identidad y cómo han pasado de ser prosumidores para convertirse en personalidades de referencia para aquellos que ven sus canales. Para ello, y mediante una metodología de carácter cualitativo-descriptiva, se ha elaborado una ficha de análisis compuesta por doce indicadores principales, los cuales arrojan datos acerca de la actividad que desempeñan en cada uno de sus vídeos, así como la exposición de las marcas. En una segunda fase del estudio, los sujetos que forman parte de la muestra serán sometidos a un análisis desde el punto de vista de su dimensión iconográfica, psicológica y sociológica, las cuales son propias de la construcción de personajes ficcionales. Los resultados obtenidos demuestran, por un lado, que las marcas corporativas no siempre están presentes en los vídeos mostrando productos propios para el público infantil y, por otro, que los youtubers analizados se autorepresentan habiéndose forjado una identidad plagada de estereotipos, los cuales, en algunos casos, transmiten actitudes y comportamientos nocivos para el público infantil.

Palabras clave: YouTube, youtubers infantiles, marcas, personajes, estereotipos.

\section{Child YouTubers: the use of minors as advertising trendsetters}

\begin{abstract}
This paper has two goals. On the one hand, it analyzes the brands featured in videos from internationally recognized child YouTubers. And on the other, it stresses the need to look at these young stars from the point of view of their identity, and to chart how they have turned from prosumers into influential personalities in the eyes of their viewers and subscribers. This second
\end{abstract}


goal is a response to the existing literature on the subject, which fails to account for how child YouTubers, due to their success, have become very much like fictional characters. To this end, we adopted a qualitative descriptive approach and settled on twelve main indicators, which offer insights on the presence of brands as well as on the activities carried out by these YouTubers in their respective videos. In a second phase of this study, the featured YouTubers will be analyzed from iconographical, psychological, and sociological perspectives, as one would pick apart the construction of a fictional character. Our results show that the brands in these videos do not always advertise products aimed at children. And as for child YouTubers, the identities they have forged for themselves are often riddled with stereotypes - which can, in some cases, convey attitudes and behaviors that are harmful to young audiences.

Keywords: YouTube, child YouTubers, brands, fictional characters, stereotypes.

\section{Youtubers infantis: menores como recurso paragerar novas tendências publicitárias}

\section{Sumário}

Considerando os trabalhos anteriores publicados por outros autores, o objetivo neste caso é duplo. Por um lado, pretende-se analisar as marcas presentes nos canais dos youtubers infantis mais reconhecidos internacionlamente; por outro lado, e como nenhuma das investigações anteriores deteve-se para estudar esse grupo como menores que, devido ao seu reconhecimento, acabaram se tornando pessoas com certa fama, este trabalho suscita a necessidade de analisá-los do ponto de vista de sua identidade e de como passaram de ser prosumers a se tornaram figuras de referência para quem assite a seus canais. Para isso, e por meio de uma metodologia qualitativa-descriptiva, foi elaborada uma folha de análise composta por doze indicadores principais, que fornecem dados sobre a atividade realizada em cada um de seus vídeos, bem como a exposição das marcas. Numa segunda fase do estudo, os sujeitos que fazem parte da amostra serão submetidos a uma análise do ponto de vista de sua dimensão iconográfica, psicológica e sociológica, característica da construção de personagens ficcionais. Os resultados obtidos mostram, por um lado, que as marcas corporativas nem sempre estão presentes nos vídeos que mostram seus próprios produtos para o público infantil e, por outro, que os youtubers analisados se representam tendo forjado uma identidade repleta de estereótipos, em alguns casos, que transmitem atitudes e comportamentos prejudiciais para as crianças.

Palavras chave: YouTube, youtubers infantis, marcas, personagem, figura, estereótipos.

\section{Introducción}

YouTube se ha convertido en la plataforma de vídeos por excelencia, llegando a contabilizar millones de visualizaciones cada día. No solo se trata de un espacio para exponer contenido audiovisual, sino que, tal y como defienden autores como Sabich y Steinberg (2017), Sánchez-Vera, Solano Fernández y Recio Caride (2019), se presenta como un escenario interactivo en el que prima la comunicación bidireccional.

$\mathrm{Al}$ igual que sucede con otros movimientos sociales localizados en un entorno determinado, en el medio digital se ha vislumbrado la existencia de líderes miembros de una nueva tribu que, en poco tiempo, se han convertido en los protagonistas de este medio, y en concreto de la plataforma YouTube. Si se tiene en cuenta que una tribu, 
Austral Comunicación

Volumen 9, número 2 (Diciembre de 2020): 249-274. ISSN 2313-9129

según la Real Academia Española, es un grupo perteneciente a un mismo origen y cuyos miembros suelen tener en común usos y costumbres, nuestro ámbito de estudio encuentra en dicha definición cierta relación. Así, se entiende a los youtubers infantiles como miembros activos de un grupo nacido en un entorno digital (mismo origen) y que utilizan la tecnología que los rodea como una extensión de sí mismos, siendo para ellos la creación y difusión de contenidos la práctica fundamental (usos y costumbres comunes). Estos sujetos, que forman parte de la tribu, se valen de sus actividades, bajo el rol de prosumers, para generar influencia en la cultura digital proponiendo nuevos modos de comunicación.

Los líderes mencionados encajan en la figura del influencer, pero esta no es reciente, pues siempre han existido personas con cierta credibilidad que han ejercido influencia sobre sus seguidores. Si se toma como base la funcionalidad de esta figura, los youtubers se identifican como sujetos que llevan a cabo la misma práctica. En este sentido, Sáez y Gallardo (2017) afirman que un youtuber es un líder de masas que, a través de su contenido audiovisual, influye sobre las actitudes y conductas de otras personas. Burgess y Green (2013), por su parte, determinan que la influencia del youtuber puede medirse por el número de suscriptores y reproducciones de los vídeos. Aunque, según Berzosa (2017) y Chau (2010), la interacción se constituye como una de las características fundamentales, ya que los comentarios realizados por los usuarios normalmente reciben una respuesta de los youtubers, lo que los hace parecer accesibles, amigables y los convierte en importantes referentes sociales (Westenberg, 2016), autores como Gallardo Camacho y Jorge Alonso (2010), Gallardo Camacho (2013) y Tur Viñes, Núñez Gómez y González Ríos (2018) opinan lo contrario. Estos afirman que la interacción en YouTube no se entiende como un factor clave, ya que observan que los prosumidores se valen de esta plataforma como una herramienta de proyección identitaria más que como un espacio de intercambio, con lo cual cuestionan el carácter social que a veces se le otorga.

Si bien los youtubers pueden ser personas de diferentes edades, quienes acceden a esta red también presentan perfiles diversos, siendo uno de los principales públicos los niños que acuden a esta plataforma en busca del entretenimiento. Como consecuencia directa, Ramos y Herrero (2016) afirman que el 69\% tienen cuentas en la plataforma, mientras que el $93 \%$ son participantes activos. Quienes producen contenido se dedican a la grabación de vídeos de forma amateur en un principio y profesional más tarde, a medida que van obteniendo mayor número de suscripciones (Rego y Romero Rodríguez, 2016), lo cual se traduce en el apoyo de las marcas comerciales y, por ende, en ganancias para ambas partes. Sobre este asunto trabajan Aznar Díaz et al. (2019), analizando la figura de los padres "que ejercen un control en la gestión de los vídeos, siendo esta controlada, en los casos de mayor éxito, por grandes compañías que buscan 
Inmaculada Sánchez, Labella Martín

Youtubers infantiles: los menores como recurso para generar nuevas tendencias publicitarias

el beneficio económico" (p. 116). En este sentido, Tur Viñes, Núñez Gómez y González Ríos (2018) critican la desprotección de la infancia y la imagen del menor, el cual no es consciente de las ganancias monetarias que está obteniendo y de las posibles repercusiones que puede tener ese estilo de vida en el desarrollo cognitivo y social a largo plazo. Un informe de Unicef (2017) indica que uno de cada tres menores en el mundo está conectado a la red y que YouTube es uno de sus sitios preferidos porque les confiere numerosos y diversos contenidos con los que entretenerse. Por su parte, la publicación MásQueNegocio ofrece datos sobre el aumento del uso de este canal, indicando un incremento de más de mil quinientos millones de usuarios, con una subida de quinientas horas de contenido de vídeo por minuto en el año 2017. El caso más reconocido es el de Ryan, del canal "Ryan's World", un niño de seis años considerado como "el miembro más joven y mejor pagado del mundo de las estrellas de YouTube con 11 millones de dólares aproximadamente", según menciona O’Connor (2017) para la revista Forbes. Por su parte, en el informe publicado por el portal eMarketer (2016) se sugiere que estos niños han de recibir el nombre de "nativos de YouTube", en vez de "nativos digitales", ya que casi las tres cuartas partes de los menores de 11 años ven vídeos digitales al menos una vez al mes.

Si se toman como referencia los datos expuestos más arriba, nos detenemos en este punto para apuntar la línea de estudio principal en la que se basa el presente trabajo: la presencia de los youtubers infantiles como personajes fundamentales de la nueva tribu digital que generan influencia y cómo estos se han convertido en transmisores de contenido publicitario.

\section{Los youtubers, una vía para que las marcas lleguen al público infantil}

Los datos arrojados por el Asociación para la Investigación de Medios de Comunicación (2018) sitúan el consumo de vídeos online en un $89 \%$ entre los niños de edades comprendidas entre los 6 y los 13 años. Así, y "a pesar de que el visionado creciente de estos contenidos por parte de los menores genera preocupación social, al mismo tiempo supone un ámbito interesante para las marcas" (Tur Viñes, Núñez Gómez y González Ríos, 2018, p. 1212). Las marcas comerciales aprovechan la interactividad y la inmediatez que proporciona el escenario digital pero, aun así, "la literatura sobre infancia y publicidad en los soportes móviles evidencian una cierta anarquía con respecto a la diferenciación y categorización de los mensajes publicitarios lanzados a través de estas plataformas" (Feijoo y Pavez, 2019, p. 315). Si bien los estudios relacionados con la infancia se han enfocado en aspectos como el uso de la tecnología (Livingstone, 2003; 2009) o los efectos sociales y psicológicos (Correa, Straubhaar y Spence 2015; Hargittai y Hinnant, 2008), "el contenido publicitario online, al que se enfrentan los menores, ha recibido menos atención” (Feijoo y Pavez, 2019, p. 314).Y es que se trata de un fenómeno 
emergente y complejo a la vez, que nace como producto de la convergencia mediática tecnológica dando paso a una cultura participativa donde se difumina el rol de productores y consumidores, en un proceso de carácter colectivo que involucra a industrias, marcas y audiencias por igual (Jenkins, 2006).

En este sentido, el trabajo realizado por Fernández Torres y Chamizo Sánchez (2020) ofrece datos interesantes, determinando que dada la repercusión que las nuevas tecnologías suponen para la sociedad, y el uso que de ellas hacen las generaciones más jóvenes, no es de extrañar el interés que este panorama puede despertar en los anunciantes que ven cómo es cada vez más difícil alcanzar a este segmento de la población a través de los canales convencionales (2020). Las marcas han comprendido que en estos escenarios los menores son los mejores influencers para promocionar sus productos y servicios (Marsh, 2016) y que, al mismo tiempo, los viewers son consumidores activos que influyen en las decisiones y procesos de compra de los adultos (Livingstone, Mascheroni y Staksrud, 2015; Raun, 2018).

Aunque parte de la intención de consumo sea el entretenimiento, estos contenidos resultan atractivos porque los menores ven a estos youtubers como un grupo de iguales. Se presentan como personas cercanas que comparten rasgos similares, produciéndose así una rápida identificación con ellos (Pérez-Torres, Pastor-Ruiz y Abarrou-Ben-Boubaker, 2018), pero estos se convierten, a su vez, en portadores de marcas comerciales y modelos a seguir que resultan atractivos por la sensación de autenticidad que transmiten, la accesibilidad de sus contenidos y la intimidad que comparten (Aran Ramspott, Fedele y Tarragó, 2018). A este respecto, y pensando en la presencia continua de marcas comerciales en este espacio, resulta interesante la afirmación de Ramos y Herrero (2016) cuando estiman que el $48 \%$ de los jóvenes de 8 a 15 años han descubierto productos en YouTube que posteriormente les piden a sus padres. Pero, como indican Tur Viñes, Núñez Gómez y González Ríos (2018) "los límites entre los contenidos no publicitarios y los que sí lo son se desdibujan en YouTube pudiendo confundir a la audiencia infantil sobre su intención final" (p. 1214). Este planteamiento se soporta en diferentes estudios, los cuales indican que los menores de 8 años tienen dificultades para identificar la publicidad en línea en comparación con la publicidad televisiva tradicional, ya que la comunicación basada en la influencia resulta más efectiva, eficiente y creíble al estar inmersa en un contenido que refleja la cotidianidad de los emisores, resultando el mensaje más natural (Blades Oates y Li, 2013; Kunkel y Castonguay, 2012; Panic, Cauberghe y Pelsmacker, 2013; Tur Viñes, Núñez Gómez y González Ríos, 2018). La peligrosidad del contenido publicitario es palpable, por ello, es necesario proveer a los viewers de pautas educativas que les permitan discernir la intencionalidad de los contenidos. En esta línea, Evans, Grubbs Hoy y Carpenter Childers (2019) plantean que "es poco probable que los padres moderen el acceso de los niños al contenido comercial o que incluso favorezcan una discusión sobre la alfabetización publicitaria" (p.2). Así, Hudders y Cauberghe 
Inmaculada Sánchez, Labella Martín

Youtubers infantiles: los menores como recurso para generar nuevas tendencias publicitarias

(2018) establecen que "estos comportamientos, definidos como 'estrategias de mediación de los padres', suponen pautas educativas que permiten a los menores lidiar con el contenido de los medios y prevenir las consecuencias negativas de su uso en su salud psicológica y mental" (p. 199).

\section{Creación y difusión de patrones de comportamiento}

$\mathrm{Al}$ ser conscientes de la función socializadora de las plataformas online, se ha de considerar que no todo lo que se emite es loable; preocupa qué se cuenta, cómo se cuenta, así como el tratamiento que se les concede a los influencers como personajes de referencia que causan efecto sobre los usuarios. Si se parte del análisis de los efectos sociales, y centrándonos en el público infantil, es fácil que los niños aprendan y aprehendan actitudes, nociones y situaciones estereotipadas que les vienen impuestas por estas figuras; este se entiende como un público vulnerable si tiene pocas guías de conducta y/o carece de experiencia relevante.

Más allá de la posibilidad de que los contenidos emitidos manifiesten, en algunos momentos, prácticas nocivas o poco apropiadas para este público, en este caso se presta especial atención a los estereotipos representados por suponer una transmisión sutil de modos de ser y hacer, los cuales pueden ser adoptados como modelos de referencia. $Y$ es que las representaciones estereotipadas socializan, ya que la audiencia encuentra en los personajes que las representan modelos de identificación y comparación que pueden repercutir en la construcción de sus valores, creencias o actitudes (Hoffner y Buchanan, 2005; Medrano y Martínez de Morentín, 2012).

La naturalidad en la que se enmarcan las peripecias de los youtubers y sus hazañas enmascaran el ápice de ficción que envuelve sus actuaciones. A pesar de que estos son menores que interpretan su guion en los sucesivos vídeos, al mismo tiempo se convierten en personajes de referencia que si bien en un inicio actuaban por placer y pura iniciativa, cada vez más lo hacen reinterpretando unas pautas bien para ofrecer a sus seguidores lo que estos demandan y esperan de ellos (aquello que los cautivó), o bien para cumplir con las exigencias por parte de las marcas comerciales que los apoyan en aras del aumento de suscriptores.

El modo de ser y hacer de los personajes, involucrados en cualquier temática, supone un acercamiento a partir del cual el usuario experimenta y reflexiona. Por ello, y más allá de las perspectivas ya expuestas, se cree necesario focalizar los estudios sobre los youtubers considerándolos como personajes que sirven de referente a los niños que consumen sus contenidos. Por este motivo, en este trabajo se plantea la importancia de controlar cómo se construyen los personajes, así como sus modos de actuación, ya que las consecuencias de sus acciones suponen un referente para la audiencia y un modo de ver la realidad. 
Austral Comunicación

Volumen 9, número 2 (Diciembre de 2020): 249-274. ISSN 2313-9129

\section{Materiales y métodos}

Como hemos explicado en el marco teórico, esta investigación parte de la necesidad de ofrecer una nueva perspectiva de análisis que amplíe las desarrolladas hasta ahora por autores que previamente se han enfrentado a esta temática. Entre los trabajos más recientes, se mencionan los de Aznar Díaz et al. (2019), León (2018), Montes, García y Menor (2018), Suing, Salazar y Ortiz (2018) y el de Tur Viñes, Núñez Gómez y González Río (2018), en los se busca identificar las prácticas de los youtubers infantiles en España, conocer el proceso de creación de los menores en YouTube, saber acerca de la influencia y relación entre los principales youtubers de América Latina, así como determinar el tipo de contenido y el apoyo que reciben de las marcas. Si se consideran estos trabajos como precedentes, el objetivo en este caso es doble: 1) Por un lado, se pretende analizar la presencia de las marcas en los canales de los youtubers infantiles más reconocidos internacionalmente; 2) por otro lado, y dado que ninguna de las investigaciones previas se detiene a estudiar a los youtubers infantiles como menores que, por su reconocimiento, han terminado convirtiéndose en personajes, este trabajo plantea la necesidad de analizar a estos niños desde el punto de vista de su identidad y cómo han pasado de ser prosumidores a convertirse en personajes de referencia para aquellos que ven sus canales. Esta intención se soporta en las palabras de Lorente (2017), las cuales determinan que el grado de influencia que presentan los youtubers sobre los menores se vincula al proceso de construcción de la identidad y al sentido de pertenencia a un grupo, incrementándose en el caso de los youtubers infantiles que son considerados por otros niños como iguales, así como el efecto que los contenidos subidos puedan tener. Del mismo modo, el planteamiento de Martínez y Olsson (2019) nos respalda al afirmar que se trata de un colectivo vulnerable que se encuentra en pleno desarrollo físico y emocional, siendo muy influenciables por el contexto, en este caso digital.

Conforme a los antecedentes expuestos, quisimos investigar la representación de los youtubers infantiles estableciendo dos hipótesis:

$\mathrm{H} 1$ : los youtubers infantiles son utilizados por las marcas comerciales como vías para formular nuevos modos de comunicación publicitaria, incluyendo en todos sus vídeos marcas de productos y/o juguetes utilizados en el día a día por el público infantil.

H2: la repercusión de los youtubers infantiles hace que estos tengan que adaptarse a su propio guion, por lo que han dejado de ser meros prosumidores para convertirse en personajes de referencia, habiéndose construido en torno a las dimensiones propias de los personajes ficcionales (dimensión iconográfica, psicológica y sociológica). 
Inmaculada Sánchez, Labella Martín

Youtubers infantiles: los menores como recurso para generar nuevas tendencias publicitarias

\section{Metodología}

El método de este artículo parte del análisis de los canales de YouTube con mayor repercusión a nivel mundial, protagonizados por niños. El estudio parte de una serie de indicadores que derivan de las necesidades expuestas en el apartado anterior. El conjunto de datos analizados se ha sometido a una metodología de carácter cualitativo-descriptivo. Así, en primer lugar, se ha procedido a una revisión bibliográfica centrada en la repercusión de las redes sociales, en concreto de la plataforma YouTube, el uso de este espacio como un canal óptimo para las marcas comerciales, así como su función socializadora. Finalmente, utilizando como herramienta de abordaje el análisis de contenido, se ha procedido a la observación pormenorizada de los canales que conforman la muestra. Para ello, el trabajo se ha dividido en dos fases.

Para atender al primer objetivo, en la primera fase de la investigación se ha confeccionado una plantilla inspirada en algunos de los ítems planteados por Segarra Saavedra e Hidalgo Marí (2018). Se trata de un instrumento compuesto por un total de doce indicadores principales con los que analizar cada uno de los vídeos publicados por estos youtubers.

\section{A) Datos generales}

1. Nombre del canal. Nos permitirá conocer si el nombre del canal tiene algún tipo de relación con su contenido, de manera que sea identificable por el usuario.

2. Fecha de creación del canal. Nos permitirá saber desde cuándo está activo el canal.

3. País.

4. Otros datos de contacto. Se tendrá en consideración si los canales ofrecen vías de contacto alternativas con las que mostrarse más cercanos a los usuarios.

5. Temática. Resulta interesante saber si en todos los vídeos se recurre a la misma temática o si, por el contrario, se abordan temas diferentes con los que atraer a los usuarios.

Atento a lo planteado anteriormente por Burgess y Green (2013), se cree necesario prestar atención a:

6. Comunidad. Número de suscriptores.

7. Visualizaciones. Conviene saber el total de reproducciones de cada vídeo con la intención de conocer el grado de atención causado en los usuarios. 
Austral Comunicación

Volumen 9, número 2 (Diciembre de 2020): 249-274. ISSN 2313-9129

B) Gestión y estrategia de contenido. Se considera elemental que los canales tengan definida y lleven a cabo una estrategia de contenido con la que ajustarse a un plan comercial y de comunicación. Para ello, tendrán validez los siguientes ítems:

8. Frecuencia de publicación. El cumplimiento de este indicador permite conocer si la cuenta se muestra cercana a la ciudadanía con la publicación de contenidos continuados. De este modo, se contribuye a la fidelización de los usuarios.

9. Marcas presentes. Nos permitirá saber si los canales analizados tienen una finalidad publicitaria gestionada mediante el entretenimiento.

10. Marcas que se publicitan antes del comienzo del vídeo. Con la misma intención perseguida en el ítem anterior, se pretende comprobar si los anuncios previos al contenido del canal tienen relación con la temática de este y si, del mismo modo, es publicidad para el público infantil.

\section{C) Uso de la plataforma YouTube}

11. Engagement. Uno de los retos más complejos en las redes sociales es generar engagement con el usuario, fidelización o compromiso de quienes siguen la cuenta. La publicación contante (indicador B8) y la interacción con el contenido constituyen la baza principal.

12. Interacción. Este indicador puede medir:

12.1. Si los usuarios comentan los vídeos y/o seleccionan la opción "me gusta"/"no me gusta".

12.2. Si existe comunicación bidireccional entre quien gestiona el canal y los usuarios. El cumplimiento de este indicador resulta primordial para cumplir con la interacción.

12.3. Igualmente, se tendrán en cuenta los tipos de comentarios, en caso de que existan.

Algunos indicadores están formulados de manera que las respuestas solo pueden ser positivas o negativas en el caso de que estas no se hayan manifestado, mientras que en otros casos las respuestas arrojan contestaciones más descriptivas, las cuales fundamentan aspectos más intrínsecos. Tanto los indicadores como el modo en que se evalúan los ítems de análisis verifican las características formales de los canales seleccionados, así como el modo en que se representan a los niños analizados.

En la segunda fase del estudio, la cual dará respuesta al segundo objetivo, los elementos de la muestra que así lo permitan serán sometidos a un análisis de persona- 
Inmaculada Sánchez, Labella Martín

Youtubers infantiles: los menores como recurso para generar nuevas tendencias publicitarias

jes de carácter cualitativo. La herramienta será una plantilla creada en 2009 para esta finalidad por el Equipo de Investigación en Análisis de Medios, Imágenes y Relatos Audiovisuales (AdMIRA, -SEJ496-) de la Universidad de Sevilla, la cual está basada en las teorías de Casetti y Di Chio (2007). Se trata de un instrumento que, a su vez, es heredero de los aportes de destacados narratólogos, como Chatman (1990), Greimas o Propp, autores fundamentales en las investigaciones de personajes audiovisuales. La plantilla atiende a una dimensión iconográfica, psicológica y sociológica, las cuales permiten conocer la apariencia, las motivaciones y las actuaciones. Además, se analizarán el rol y los estereotipos representados sobre los que se construyen los personajes. Estos factores, empleados como indicadores en el análisis, verifican que todo personaje viene definido por su ser (identidad, rasgos físicos, carácter, etc.), por su hacer (en este caso, cómo los youtubers exponen el discurso) y por la conducta que desarrollan (Sánchez, 2000, p. 126). Con ello, se pretende apostar por el análisis de unos niños youtubers que se han construido como personajes sometidos a una narratología previamente definida.

Los datos recogidos se trabajaron con el programa Microsoft Excel, con cuyas hojas de cálculo se pudo efectuar una serie de operaciones. Por un lado, el análisis realizado en la primera fase, con respecto a los ítems referidos a cuestiones discriminatorias, se basa en el sistema binario de ceros y unos para indicar si se dan o no cada uno de los indicadores en los canales analizados. Por otro, y centrados en la segunda fase del estudio, mediante palabras clave se desarrollarán breves descripciones que nos permitirán conocer las dimensiones iconográficas, psicológicas y sociológicas de cada niño, extrayendo un perfil general.

\section{Muestra}

Para la selección de la muestra, se tomaron como válidos los canales de los youtubers infantiles con más seguidores a nivel internacional. Para ello, se ha confeccionado un listado considerando los datos más actuales: la publicación de la web marketing4ecommercet.net, la noticia lanzada por el diario español La Vanguardia, así como los perfiles analizados por Tur Viñes, Núñez Gómez y González Río (2018), más próximos a nuestro estudio y basados en la base de datos SocialBlade.

Estos son: "Ryan's World”, "Its JoJo Siwa", “Gibby", "SIS vs BRO”, “TV Ana Emilia”, "MikelTube", "Los Juguetes de Arantxa”, "EthanGamer", "Darwin”, “JillianTubeHD”, "CharlisCraftyKitchen", "Vicente Espinosa", "Las Ratitas", "Juega con Adri”, "La diversión de Martina", "Los Mundos de Nico" y "Juguetes Maryver".

El periodo analizado abarca el último trimestre del año 2019 (desde el 1 de octubre hasta el 31 de diciembre). El motivo de elección de este tramo no es otro que el de buscar la mayor cercanía temporal con el análisis de datos, para que las conclusiones 
obtenidas sean lo más actuales posibles. La realización del estudio se llevó a cabo en los meses de enero a marzo de 2020.

\section{Discusión de resultados}

Al tomar en cuenta que si bien el análisis aquí realizado no es determinante, puesto que se limita al último trimestre del año 2019 y se ciñe exclusivamente a los canales más vistos según las fuentes de datos anteriormente mencionadas, se debe entender como un trabajo que complementa y enriquece la literatura existente. De este modo, se contribuye a proporcionar más información acerca de los youtubers infantiles.

Antes de proceder a la lectura de resultados, al igual que apuntan otros trabajos, se ha de comentar que las cifras de followers y visualizaciones de los canales oscilan constantemente. Es por ello que a pesar de que las publicaciones en las que se basa este trabajo mencionan el canal "Darwin" como uno de los más influyentes, se observa que en el momento en que se desarrolló el presente estudio este no se encontraba disponible en YouTube. Aprovechamos para comentar que, igualmente, los canales "CharlisCraftyKitchen" y "Vicente Espinosa" no serán considerados como parte de la muestra, ya que el contenido disponible es obsoleto y no se ajusta a la franja temporal determinada. Así, y sabiendo que la muestra queda limitada a catorce canales, a continuación, se procede al comentario de los datos obtenidos.

Según el orden de indicadores expuesto en la ficha de análisis y centrados en los datos generales, los resultados obtenidos demuestran que el $71 \%$ de los canales son de fácil localización, ya que emplean como nombre identificativo el nombre propio del niño, lo cual apunta hacia el protagonismo del youtuber en los contenidos publicados ("Ryan's World", "Its JoJo Siwa" -su nombre completo es Joelle Joanie Siwa-, "Gibby", “TV Ana Emilia," “MikelTube”, “Los Juguetes de Arantxa," "EthanGamer," "JillianTubeHD”, “Juega con Adri", "La diversión de Martina" y "Los Mundos de Nico"). En el caso del canal "Juega con Adri", se ha de precisar que la protagonista es la madre de la niña llamada Adri. Por su parte, los canales "SIS vs BRO", "Las Ratitas" y "Juguetes de MaryVer", del mismo modo, utilizan nombres llamativos, pegadizos y de fácil memorización.

En cuanto a la fecha de creación de los canales, se puede decir que oscila entre el año 2010 (en el caso del canal "Juguetes MaryVer") y el año 2018 (canal "Juega con Adri”), observándose que el grueso de canales coincide en el año 2015, tal y como indicó en su trabajo Aznar Díaz et al. (2019, p. 118). Del lugar de origen se realiza el mismo comentario, ya que existe gran diversidad de procedencias.

Por otro lado, y atendiendo a la disponibilidad de los canales, el 100\% ofrece diferentes vías de contacto, siendo estas: dirección de correo electrónico para consultas comerciales (este dato ya vaticina la intención de la relación canal-marcas comerciales), perfiles en distintas redes sociales (Facebook, Twitter, Instagram y Tik Tok), así como 
Inmaculada Sánchez, Labella Martín

Youtubers infantiles: los menores como recurso para generar nuevas tendencias publicitarias

su presencia en blogs e incluso, en algunos casos, la creación de sus propias páginas web, lo cual indica la dimensionalidad referida a la preponderancia y la repercusión del canal y, por ende, de su protagonista.

Llegados a este punto, se han de comentar las temáticas propuestas por cada canal. El 71\% de casos presentan un contenido variado, el 7\% son canales exclusivos de videojuegos ("EthanGamer"), destinándose el $21 \%$ restante a canales que son monotemáticos. Este es el caso de "Its JoJo Siwa", donde la joven expone todo lo relativo a su entorno personal y su vida privada en una gestión del contenido que roza el género del reality. Por su parte, se ha de mencionar el canal "Las Ratitas", donde exclusivamente se trata el tema de las princesas (niñas que se dedican a maquillarse, a hacerse la manicura, muestran el gusto excesivo por el color rosa, los vestidos, los zapatos de tacón...), el canal "La diversión de Martina", dedicado en su totalidad a transmitir su vida a modo de diario (vivencias personales, su participación en series y películas de actualidad, la promoción de sus propios productos, etc.) y, en la misma línea, el canal “JillianTubeHD", en el que la youtuber, a pesar de los pocos vídeos publicados entre el mes de octubre y diciembre de 2019, muestra sus actividades como celebrity (aparece, por ejemplo, practicando el doblaje de series de dibujos animados). Los espacios que optan por el multicontenido son aquellos en los que los niños realizan actividades en el exterior, acompañados a veces por adultos (ya sea en casa, fuera de esta o durante algún viaje organizado). En este caso. proponen retos, realizan experimentos, simulan actividades propias de los adultos y practican el unboxing.

Luego de este revelamiento, estamos en disposición de corroborar las palabras de Aznar Díaz et al. (2019, p. 123) cuando afirman la existencia de estereotipias de género representadas a través de los niños, en este caso, y, en ocasiones, de la mano de marcas anunciadas. Esta afirmación se soporta en el canal de "Los Juguetes de Arantxa" (donde, a pesar de que en algunos vídeos juega a ser científica, en la mayor parte de ellos Arantxa reproduce acciones propias de una ama de casa desde un planteamiento estereotipado: juega a ser mamá, a ser vendedora, a enarmorarse...), en "Las Ratitas" (por todo lo anteriormente comentado: representan un mundo idílico, de cuento de hadas, siempre rodeadas de juguetes utilizados en su mayoría por niñas: muñecas, disfraces de princesas, castillos de juguete) y en el caso de "La diversión de Martina", donde, a lo largo de sus publicaciones, da pautas para convertirse en una e-girl de moda, con todo lo que ello conlleva: uso excesivo de maquillaje, sesiones de peluquería y el gusto por la moda y las compras.

Finalmente, se ha de comentar el elevado número de suscriptores y visualizaciones de los canales analizados, lo cual determina la influencia que los contenidos difundidos pueden tener. A pesar de las elevadas cifras en ambos indicadores, el canal "Juguetes de MaryVer" reúne los datos más insignificantes en cuanto a las suscripcio- 
nes (251.000 suscriptores en el momento en que se realizó el análisis). En este sentido, se cree conveniente recurrir a la herramienta Google Trend, la cual da cuenta sobre la influencia de los youtubers en los diferentes países. Atento al periodo de análisis marcado para nuestro estudio, a continuación, se indican las principales regiones (hasta cinco) donde son más influyentes, por orden descendente y según la disponibilidad de datos. "Ryan's World": Irlanda, Emiratos Árabes Unidos, Estados Unidos, Catar y Filipinas; "Its JoJo Siwa": Canadá, Australia, Estados Unidos y Reino Unido; "Gibby": Honduras, México, Guatemala, Ecuador, Perú; "SIS vs BRO”: Australia, Baréin, Emiratos Árabes Unidos, Trinidad y Tobago; “TV Ana Emilia”: Honduras, Guatemala, Ecuador, Nicaragua y Venezuela; "MikelTube": España, Venezuela, Paraguay, Argentina y Uruguay; "Los Juguetes de Arantxa": España, Perú, Venezuela, Uruguay y Argentina; "EthanGamer": Canadá, Reino Unido, Australia y Estados Unidos; "Jillian Tube HD": Estados Unidos; "Las Ratitas": España, Bolivia, Honduras, Venezuela y Uruguay; "Juega con Adri”: Bolivia, Perú, Ecuador, Venezuela y Colombia; "La diversión de Martina": España, Argentina, Chile, México y Perú; y "Los Mundos de Nico": España, Uruguay, Venezuela, Argentina y Perú. Del canal "Juguetes MaryVer" no se ofrecen datos (ver Tabla 1).

Como parte del segundo bloque de análisis, nos detenemos a reflexionar sobre los datos obtenidos del indicador "frecuencia de publicación", "marcas presentes" y "marcas que se publicitan antes del comienzo del vídeo".

Si bien en el último trimestre de 2019 el 35\% de los canales publican de manera puntual ciñéndose a un planning previamente marcado ("Ryan's World" con treinta publicaciones al mes; "MikelTube" con ocho; "SIS vs BRO”, "Los Juguetes de Arantxa” y "La diversión de Martina" con cuatro), el 64\% no lo hace de un modo tan escrupuloso: "Its JoJo Siwa" publica de media entre 10 y 20 vídeos por mes. En el caso de "Gibby", encontramos únicamente un vídeo que encaja dentro de nuestra horquilla de análisis; "TV Ana Emilia": entre cuatro y cinco por mes; "EthanGamer": cinco por mes; "JillianTubeHD": uno por mes; "Las Ratitas": entre dos y tres por mes; "Juega con Adri": entre ocho y catorce por mes; "Los Mundo de Nico": entre ocho y nueve por mes; y "Juguetes de MaryVer": entre uno y dos por mes.

En este sentido, se observa la falta de una estrategia de contenido, dado que la mayor parte de la muestra no es constante, lo cual puede suponer un aspecto negativo para los seguidores del canal ya que no tienen constancia de cuándo tendrán disponible un nuevo vídeo. A pesar de ello, y salvo en los casos en los que se observa una distancia mayor entre publicaciones, se puede afirmar que hay una buena gestión en aras del engagement (indicador que será analizado en el siguiente bloque).

Si nos centramos en las marcas que están presentes en los distintos canales, podemos afirmar que los niños, bajo el rol de youtuber, se han convertido en espacios publicitarios 
Tabla 1. Datos generales

\begin{tabular}{|c|c|c|}
\hline Nombre del canal & Marcas que se anuncian & Marcas que anteceden al vídeo \\
\hline Ryan's World Comerciantes & Presencia no constante de marcas Iron Man, Coca-Cola y Mentos. & Juegos online infantiles "Fun Match Game". \\
\hline Its JoJo Siwa & $\begin{array}{l}\text { Tesla, BMW, Adidas, Nickelodeon Game con sus propios juegos: } \\
\text { "Nickelodeon JoJo Siwa BustABow Dance Game", muñecas } \\
\text { de su propia marca ( } 27 \text { variedad. JoJo Siwa Dolls). }\end{array}$ & \\
\hline \multicolumn{3}{|l|}{ Gibby } \\
\hline SIS vs BRO & $\begin{array}{l}\text { Apple, PlayStation, XBox, Tiny Lote Gymini (juguete de bebé), } \\
\text { Nike en vestimenta de Karina, Fisher Price y Vtech. }\end{array}$ & $\begin{array}{l}\text { Marcas diversas no siempre } \\
\text { relacionadas con el público infantil. }\end{array}$ \\
\hline TV Ana Emilia & $\begin{array}{l}\text { Presencia no constante de marcas. Apple. Pandora, } \\
\text { FujiFilm insta mini 9, taf (zapatillas deporte). }\end{array}$ & $\begin{array}{l}\text { Marcas diversas no siempre } \\
\text { relacionadas con el público infantil. }\end{array}$ \\
\hline MikeiTube & $\begin{array}{l}\text { Breakout Beast de Mattel (huevos), Bakugan (indican que } \\
\text { es patrocinio, con lo cual se interpreta que el niño hace de } \\
\text { presentador del producto), Fantasy Riders, Pokemon, Avión } \\
\text { PinyPon Actions, Super Zing, Star Wars, Actimel Danone. }\end{array}$ & \\
\hline Los Juguetes de Arantxa & $\begin{array}{l}\text { Menciona el nombre de las marcas: MyMy City, Gogo Galaxy, } \\
\text { Pulid Pocket, Lego, Besuco, Bellies, Bebe Rebom. }\end{array}$ & \\
\hline EthanGamer & Marcas de distintos videojuegos. & $\begin{array}{l}\text { Marcas diversas no siempre } \\
\text { relacionadas con el público infantil. }\end{array}$ \\
\hline \multicolumn{3}{|l|}{ JillianTubeHD } \\
\hline Las Ratitas & $\begin{array}{l}\text { Muñecas Shimmer and shine, Mattel Barbe). } \\
\text { Feber con el CastiHo de Fraseo } 2 .\end{array}$ & \\
\hline Juega con Adri & $\begin{array}{l}\text { Feber, Hacendado, Puppy Surprise, Muñecas } \\
\text { Hairdorables, Fanta Nenuco, Pintarolas. }\end{array}$ & $\begin{array}{l}\text { Marcas diversas no siempre } \\
\text { relacionadas con el público infantil. }\end{array}$ \\
\hline La diversión de Martina & Glow Martina D'Antiochia (perfume). & $\begin{array}{l}\text { Marcas diversas no siempre irneflaacntioi, } \\
\text { nmadorpocposn pelaprtyúbylic o Danonino. }\end{array}$ \\
\hline Los Mundos de Nico & $\begin{array}{l}\text { NIKE (presente en el vestuario de Nico y su padre), } \\
\text { "Una casa de libros" (serie de TV y libros). Coca-Cola, } \\
\text { Mentos, DragonBall, Lego. Star Wars. Actimel. }\end{array}$ & $\begin{array}{l}\text { Marcas diversas no siempre } \\
\text { relacionadas con el público infantil. }\end{array}$ \\
\hline Los Juguetes MaryVer & $\begin{array}{l}\text { ViPoo Spray WC. Japan Candy Box, marca de } \\
\text { videojuegos (Roblox), Nadurines (marca de muñecas), } \\
\text { Nadurines Juega y Mima el planeta Gameplay. }\end{array}$ & \\
\hline
\end{tabular}

per se. En torno a esta tendencia, se ha de reflexionar acerca de la presencia de determinadas marcas. En primer lugar, cabe comentar que, en primera instancia, se prevé que las marcas publicitarias que vayan a estar presentes en estos canales serán propias para el público infantil, en cambio, se ha observado a veces la presencia de publicidad no asociada al target que tratamos en este trabajo. Se menciona así el vídeo subido por "Juguetes de MaryVer" titulado "VIPOO Spray WC Parodia en Juguetes MaryVer" en el que está presente la marca Air Wick VIPoo Spray WC (ambientador para inodoro). Este 
Austral Comunicación

Volumen 9, número 2 (Diciembre de 2020): 249-274. ISSN 2313-9129

es un ejemplo claro del peso de las marcas en estos espacios, las cuales se aprovechan de su hegemonía para estar presentes y aumentar así sus ventas.

$\mathrm{Al}$ analizar el contenido, se observa, primeramente, que la relación del producto con el perfil del protagonista del canal resulta compleja, por lo que se detecta un contenido forzado que roza el absurdo, en el que ni la propia protagonista resulta creíble, y todo ello fraguado para que la marca tenga cabida en la sintonía del canal. Para conseguir el ajuste, han construido una situación de parodia en la que Irene, junto con su hermano, vierten al inodoro dulces de chocolate a modo de heces para demostrar la efectividad del producto. Resulta curioso que los dulces son propios de la marca Donettes, muy reconocida para el público infantil en España. Una situación similar ocurre en el canal "Juega con Adri”. En él, marcas como Fanta (bebida refrescante de naranja de la compañía Coca-Cola), chocolate M\&M y otras -como Pintarolas (marca de chocolates de Mercadona, una conocida cadena de supermercados en España) o la marca Gelli Baff (gelatina para baños) - están presentes en dicho canal. Pero ¿cómo hacer que el público infantil consuma estas marcas que en un principio no son exclusivas de este target? Para ello, se crean contenidos que resulten divertidos y atractivos. Así, quienes construyen el guion de este canal de YouTube deciden llevar a cabo actividades estridentes e incluso exageradas, siendo algunas de ellas pícaras travesuras: llenar la bañera con Fanta, con chocolatinas M\&M o gelatina (vídeos "Nos bañamos en 1000 litros de Fanta" -2,3M visualizaciones-, "Llenamos mi bañera de M\&M Chocolate" $-5,4 \mathrm{M}$ visualizaciones- $\mathrm{y}$ "Llenamos mi bañera de espuma y gelatina" $-3,7 \mathrm{M}$ visualizaciones-). Lo mismo sucede en el canal "Ryan's World", donde aparecen marcas como Coca-Cola y Mentos, o en el canal "TV Ana Emilia”, donde la presencia de las marcas Apple o Pandora copan algunos de sus vídeos.

Más allá de estos ejemplos, en el resto de los canales las marcas resultan adecuadas para el público infantil. En este caso, y subrayando de nuevo la fuerza del aspecto publicitario para la subsistencia de estos canales, se ha de precisar que, ante el objetivo de visibilizar la marca, se opta por crear una historia entorno a ella. Entendemos esta intención como una estrategia publicitaria basada en la práctica del brand content.

Inmersos en el ámbito de la publicidad, resulta de interés prestar atención a aquellas marcas que están presentes en espacios publicitarios antes del comienzo del vídeo. Se trata de un posicionamiento estratégico, ya que el niño que se disponga a consumir el contenido del canal previamente tendrá que ver la marca hasta el momento en el que el propio anuncio le ofrece la opción skip. Esto es válido para aquellos que, con un cierto grado de conocimiento, pueden optar por no consumir en su totalidad el anuncio. En cambio, quienes no tienen dicha capacidad, suponen un público de interés, pues consumirán el spot en su totalidad. Así, se sabe que tan solo al 7\% de los canales le anteceden anuncios basados en marcas de juguetes o cualquier otro tipo de producto 
para el público infantil. Este es el caso del canal "Ryan's World", con publicidad previa de juegos online infantiles (Fun Match Game, en este caso). En el resto, cuando existen, las marcas son diversas, apareciendo, en alguna ocasión, aquellas que son para el público infantil. En el canal "EthanGamer", el contenido es exclusivo de diversas marcas de videojuegos. Además, hemos de comentar que, en algunos casos, antes de que dé comienzo el vídeo, se indica que se trata de un contenido comercial. Finalmente, el canal "La diversión de Martina" resulta interesante, ya que en él se exponen marcas propias y productos que se han creado para la protagonista.

Tabla 2. Marcas publicitarias presentes en los canales

\begin{tabular}{|c|c|c|c|c|c|c|}
\hline Nombre del canal & Fecha & País & Datos de contacto & Temática & $\begin{array}{l}\text { Número de } \\
\text { suscriptores }\end{array}$ & $\begin{array}{l}\text { Número de } \\
\text { visualizaciones }\end{array}$ \\
\hline Ryan's World & 2015 & EEUU & $\begin{array}{l}\text { Correo electrónico } \\
\text { y redes sociales }\end{array}$ & Variada & $24,2 \mathrm{M}$ & 36.902 .751 .515 \\
\hline Its JoJo Siwa & 2015 & EEUU & $\begin{array}{l}\text { Correo electrónico, redes } \\
\text { sociales y web propia }\end{array}$ & Vida personal & $10,7 \mathrm{M}$ & 2.853.056.713 \\
\hline Gibby & 2014 & México & Redes sociales & Variada & $10,6 \mathrm{M}$ & 2.116.170.557 \\
\hline SIS vs BRO & 2016 & Canadá & $\begin{array}{l}\text { Correo electrónico, redes } \\
\text { sociales para la niña } \\
\text { (Karina) y para el niño } \\
\text { (Ronald), web propia para } \\
\text { vender sus productos }\end{array}$ & Variada & $12.1 \mathrm{M}$ & 5.142.347.279 \\
\hline TV Ana Emilia & 2014 & México & $\begin{array}{l}\text { Correo electrónico y } \\
\text { redes sociales Variada }\end{array}$ & Variada & $8.28 \mathrm{M}$ & 2.273.980.296 \\
\hline MikeiTube & 2015 & España & $\begin{array}{l}\text { Correo electrónico, redes } \\
\text { sociales y dirección } \\
\text { postal personal. }\end{array}$ & Variada & $5.44 \mathrm{M}$ & 3.336.356.186 \\
\hline Los Juguetes de Arantxa & 2015 & España & $\begin{array}{l}\text { Correo electrónico y } \\
\text { dirección postal personal }\end{array}$ & $\begin{array}{l}\text { Variada (enfocada en } \\
\text { "juegos de niñas") }\end{array}$ & $3,3 \mathrm{M}$ & 1.123.543.409 \\
\hline EthanGamer & 2013 & UK & $\begin{array}{l}\text { UK Correo electrónico, } \\
\text { dirección postal, redes } \\
\text { sociales y dirección postal }\end{array}$ & Videojuegos & $2,57 \mathrm{M}$ & 1.847.607.181 \\
\hline JillianTubeHD & 2013 & EEUU & $\begin{array}{l}\text { Correo electrónico y } \\
\text { Redes sociales }\end{array}$ & Enfocada en sí misma & $1,52 \mathrm{M}$ & 385.096 .442 \\
\hline Las Ratitas & 2015 & España & $\begin{array}{l}\text { Correo electrónico y } \\
\text { Redes sociales }\end{array}$ & Temática princesas & $18,6 \mathrm{M}$ & 6.078 .473 .661 \\
\hline Juega con Adri & 2018 & España & $\begin{array}{l}\text { Correo electrónico y } \\
\text { Redes sociales }\end{array}$ & Variada & $2,35 \mathrm{M}$ & 566.753 .509 \\
\hline La diversión de Martina & 2015 & España & $\begin{array}{l}\text { Correo electrónicoLa redes } \\
\text { sociales y web propia }\end{array}$ & Enfocada en sí misma & $3.9 \mathrm{M}$ & 867.486 .984 \\
\hline Los Mundos de Nico & 2013 & España & $\begin{array}{l}\text { Correo electrónicoLa redes } \\
\text { sociales y web propia }\end{array}$ & Variada & $2,44 M$ & 560.687 .977 \\
\hline Los Juguetes MaryVer & 2010 & España & Redes sociales y blog & Variada & 251.000 & \\
\hline
\end{tabular}

Fuente: elaboración propia. 
El modo de gestionar el contenido, ya sea publicitario o no, resulta clave para la generación del engagement. Tal y como hemos comentado más arriba, a pesar de que en algunos canales la publicación de contenido oscila en el tiempo, se puede decir que en el $92 \%$ de los casos se produce el engagement. En cuanto a la interacción, el total de la muestra recibe "me gusta" por parte de los usuarios. Aun así, se ha de precisar que en el caso del canal "JillianTubeHD” el número de likes resulta bajo en comparación con el resto de los canales.

Como parte de la interacción, dado que el contenido que trabajamos está publicado en un medio digital, el cual ofrece herramientas para la multidireccionalidad de la información, hemos de prestar especial atención a estos canales en tanto y en cuanto se crean para generar la participación entre emisor y receptores. Como se ha podido comprobar, y a pesar de que los seguidores interactúan con los youtubers haciendo clic en las opciones like/dislike, el $85 \%$ de los canales tienen desactivada la posibilidad de realizar comentarios, lo cual afirma la ausencia de comunicación bidireccional. Tan solo en el caso de "Juega con Adri" se ofrece la posibilidad de realizar comentarios e intercambiar información; a pesar de ello, el canal no responde. Así, el objetivo de la interacción queda en parte invalidado y se entiende que, aunque estos niños están presentes en YouTube, no hacen un uso debido del canal. Estos datos corroboran lo planteado por Gallardo Camacho y Jorge Alonso (2010), Gallardo Camacho (2013) y Tur Viñes, Núñez Gómez y González Ríos (2018).

Con la interpretación de todos estos datos, y conociendo el elevado número de suscriptores, visualizaciones e interacción -likes y dislikes-, se deduce que estos jóvenes son figuras de referencia para su público, conformando parte de la nueva tribu digital; su modo de ser y hacer ha constituido una nueva tendencia en el ámbito online. Se sabe que más allá de crear historias por y para las marcas, o de protagonizar cualquier otro tipo de contenido, estos jóvenes han pasado de desempeñar el rol de prosumidores al de prescritores -al recomendar sus vídeos mediante frases hechas que incitan a ello, tal y como ya indican Tur Viñes, Núñez Gómez y González Ríos (2019)-, convirtiéndose, además, en personajes. Independientemente de la temática del vídeo, los youtubers estudiados están sujetos a una manera de representarse que, aunque con pequeños matices, debe mantenerse constante con la intención de seguir agradando y alcanzar así la fidelización de su público.

Finalizada la primera fase del estudio, y más allá de los datos expuestos, el análisis realizado nos ha permitido constatar que los youtubers infantiles que conforman la muestra presentan características propias de los personajes creados para la ficción. Así, estos se consideran aptos para ser analizados desde un punto de vista iconográfico, psicológico y sociológico (dimensiones propias en la creación de personajes). Nuestra 
Inmaculada Sánchez, Labella Martín

Youtubers infantiles: los menores como recurso para generar nuevas tendencias publicitarias

intención para aportar esta variante al estudio de los youtubers infantiles como personajes se constata.

Imbricados, pues, en la segunda fase del estudio, tenemos que advertir que, aunque cada niño youtuber se construye en torno a personajes que dinamizan temáticas diferentes, todas ellas se aproximan al entretenimiento.

Para comenzar, es necesario señalar que, en la mayoría de los vídeos -excepto en el caso se "EthanGamer", donde no se percibe de un modo tan explícito-, se crean historias breves (diez o quince minutos de media, aproximadamente), con introducción, nudo y desenlace que persuaden al público al que se dirige por la cercanía y actualidad que suponen para ellos los temas tratados: aventuras, retos, simulación de actividades propias de los adultos más cercanos y/o unboxing.

A continuación, se describe el perfil de los personajes protagonistas según las dimensiones propuestas.

En lo referido a la iconografía, hemos de destacar que todos ellos comparten características comunes. Si bien la mayor parte de los protagonistas de cada canal (71\%) tiene una edad que ronda entre los 8 y 11 años, aproximadamente, el $21 \%$ corresponde a canales de youtubers femeninas en torno a los 14-16 años. Estos datos resultan interesantes, ya que los que representan la horquilla de menor edad son tanto niños como niñas que tratan diversidad de temas, en cambio, es curiosa la ausencia de youtubers masculinos entre quienes encajan en la segunda franja (14-16 años). En este caso, son niñas que se presentan tratando exclusivamente temas propios de la adolescencia femenina: el gusto por ser reconocida, el éxito de la fama y como este le reporta al personaje la fuerza del self y la exaltación del ego, muy bien acentuados en la representación del canal "Its JoJo Siwa" y "La diversión de Martina", respectivamente. En el caso del canal de "SIS vs BRO", la protagonista de los vídeos es Karina (niña adolescente) acompañada siempre de su hermano Ronald, pero, en este caso, los temas son variados sin caer en temáticas estereotipadas según el género del personaje. Por su parte, el canal "Juega con Adri” es protagonizado por la madre de la niña (Adri). Aunque la pequeña tiene presencia en los vídeos, esta se representa adoptando un papel más secundario y poco atractivo para su público. Luego de analizar esta muestra, se entiende que es la madre quien supone el foco de atracción para sus seguidores. En este momento, conviene puntualizar que en los canales en los que los niños son los protagonistas, a veces aparecen acompañados de sus padres, concibiéndose estos como personajes secundarios. Se entiende que este acompañamiento avala de algún modo la actividad a desarrollar, proporcionando el visto bueno de las figuras parentales.

En cuanto al aspecto físico y vestimenta, se puede decir que si bien la mayor parte de ellos se representan con un estilo casual, resulta destacado cómo las protagonistas de "Its JoJo Siwa", “TV Ana Emilia”, "Los Juguetes de Arantxa”, "Las Ratitas" y "La diver- 
Austral Comunicación

Volumen 9, número 2 (Diciembre de 2020): 249-274. ISSN 2313-9129

sión de Martina" muestran un nexo en común: todas son youtubers niñas que se representan con un aspecto muy cuidado rozando los estereotipos femeninos, lo cual puede repercutir en su público. Asimismo, se ha observado cómo las protagonistas de estos vídeos muestran aspectos más cuidados que el de sus amigas cuando estas aparecen en el vídeo acompañándolas, destacándose así frente a las otras niñas. En este sentido, si bien no se critica la potenciación de la feminidad, sí se hace una reflexión profunda sobre cómo determinados modos de representación acentúan la diferencia de género.

El caso más llamativo es el de "Las Ratitas". Se trata de dos hermanas que, más allá de ser utilizadas por las marcas para visibilizar sus productos y recrear historias, a veces muestran una actitud inconformista que se subsana proveyéndolas de más juguetes. Desde una óptica educativa, hemos de detenernos en este sentido, ya que transmiten un modo de ser y hacer poco apropiado. Como personajes, representan continuamente sus vivencias en un mundo de princesas, siendo esta la principal temática. Tanto es así que la mayor de las hermanas tiene rasgos de princesa de cuento de hadas: es rubia, pelo largo, ojos grandes azules y es quien lidera las historias y quien suele tener mayores ventajas con respecto a la otra niña. Un ejemplo de ello se observa en el vídeo "Frozen 2, Las Ratitas Gisele y Claudia" (9,7M visualizaciones), en el que Gisele se viste de princesa con el mejor vestido, pareciéndose a la protagonista de Frozen, mientras que su hermana pequeña no logra sentirse princesa a pesar de probarse diferentes disfraces. En los restantes casos antes mencionados, aunque no de un modo tan acentuado, igualmente se reproducen escenas o situaciones en la misma línea: gusto excesivo por el maquillaje, niñas con manicura perfecta y reproducción de actividades consideradas, desde la óptica del androcentrismo, como propias de la mujer (jugar a ser mamá, a cuidar de la casa, a hacer la compra, gusto excesivo por la moda...).

En el caso de "La diversión de Martina", se puntualiza que su protagonista se ha convertido en un personaje reconocido fuera de las fronteras de YouTube (así lo explica en el apartado "Información" de su canal). En sus vídeos, muestra cómo ha llegado a interpretar películas de directores de cine conocidos (como Padre no hay más que uno, dirigida por Santiago Segura, 2019), convirtiéndose, al mismo tiempo, en cantante (disco Emociones) y creadora de su propio perfume (Glow Martina D'Antiochia, pour femme).

Insistimos en este momento que a priori este enfoque no denota peligrosidad alguna para el público que visita estos canales, pero no es menos cierto que todas ellas potencian los estereotipos por los que, actualmente, multitud de mujeres luchan por romper. Desde una perspectiva crítica, la reproducción de este tipo de prácticas normaliza tales actividades como "propias de la mujer". A pesar de ello, no debemos pasar por alto, en el caso de "Los Juguetes de Arantxa", la existencia de un vídeo en el que la protagonista juega a ser científica con una amiga (vídeo "Las científicas Arantxa y 
Inmaculada Sánchez, Labella Martín

Youtubers infantiles: los menores como recurso para generar nuevas tendencias publicitarias

Zaira descubren un meteorito misterioso"), pero este es un caso puntual que no supone una tendencia.

En cuanto al aspecto físico, se presta atención al canal "Juguetes de MaryVer", donde Irene, su protagonista, rompe con el patrón descrito anteriormente ya que aparece como una niña con un aspecto menos cuidado, lo cual crea un ambiente más natural y espontáneo que en los casos anteriores.

Por otro lado, un aspecto que no debemos olvidar en este punto del análisis es el tono de voz de los youtubers. Por lo general, coincidimos con Tur Viñes, Núñez Gómez y González Río (2018) al afirmar que el tono empleado es elevado y enfático, lo cual denota un efecto antinatural. En el caso de "Las Ratitas", "La diversión de Martina" y "Juega con Adri" resulta incluso estridente, pudiendo incomodar al usuario.

Como personajes, hemos de comentar el escenario en el que se mueven habitualmente. Todos los vídeos que conforman la muestra de análisis son considerados vídeos domésticos, aunque sometidos a una fase de pre y posproducción. Por lo general, los espacios en los que discurren las historias son cercanos a los protagonistas: se representa tanto el exterior como el interior de sus propias casas o locaciones del entorno habitual de cada uno de ellos, lo cual otorga mayor veracidad a las historias. En el caso de "Las Ratitas", se critica el excesivo uso del color rosa en la mayoría de los espacios, acrecentando el apunte anterior sobre la perpetuación de estereotipos femeninos.

En cuanto a la dimensión psicológica, todos representan un modo de actuación acorde a su modo de ser y comportarse. Los youtubers analizados -salvo el caso del canal "EthanGamer", quien se muestra tranquilo y persistente- representan un perfil similar: son extrovertidos, alegres, dinámicos, creativos, persuasivos, activos, entusiastas e inventivos. Estas características resultan adecuadas para captar la atención y conseguir la fidelización, pero, al mismo tiempo, supone un handicap ya que naturalmente el aspecto emocional de los niños resulta variable en el día a día, por lo que, independientemente del estado de ánimo con el que se encuentren, deben tener la capacidad de grabar cada vídeo interpretando las pautas que los definen como personajes; y es que su público, al igual que sucede con los fanes de actores, actrices, artistas y deportistas, entre otros, siempre esperan de ellos la actitud que los cautivó. Esta situación supone cierto sometimiento para estas niños, que deben adaptarse y reinterpretar continuamente sus guiones. El hecho de tener que publicar contenido de manera constante (así se demostró anteriormente) los obliga a ello si pretenden ser reconocidos y aumentar, por ende, el número de followers.

Todo este entramado hace que nos planteemos los objetivos y metas de estos como personajes, y es que todo se sustenta en el éxito del canal, del que depende, al mismo tiempo, el éxito publicitario. Así, el objetivo de cada uno de ellos radica en continuar ofreciendo contenidos que resulten atractivos para los usuarios. Si en un principio se 
Austral Comunicación

Volumen 9, número 2 (Diciembre de 2020): 249-274. ISSN 2313-9129

buscaba el entretenimiento, ahora se entiende que la presencia de los youtubers busca el rendimiento económico. Lo que en un inicio podría suponer una actividad divertida, ha terminado convirtiéndose en un trabajo cuasi profesional, perdiendo, en muchos casos, la identidad originaria del canal y de quien lo protagoniza.

Desde un punto de vista sociológico, resulta necesario resaltar la ausencia de conflictos externos de los youtubers, salvo la necesidad imperiosa de continuar aumentando el número de seguidores y visualizaciones.

El ámbito digital, escenario imperioso en la sociedad actual, se encuentra plasmado de tendencias lideradas por figuras reconocidas. En este caso, y tras la obtención de todos los datos aportados, se entiende que los youtubers que más influencia ejercen, dado el elevado número de suscriptores y visualizaciones, se han convertido en personajes reconocidos y, al mismo tiempo, en líderes de la tribu digital que conforma el panorama de YouTube, ejerciendo, además, como influencers.

Para finalizar el análisis de los youtubers como personajes, se ha observado que, en algunos casos, están sometidos a la representación de roles y estereotipos. En relación con los primeros, están vinculados con la figura del prosumidor y prescriptor, convertidos en personajes y desempeñando funciones estereotipadas. Nos hemos encontrado con canales de menores que exclusivamente se vinculan a los videojuegos, adoptando el rol de gamer ("EthanGamer") y niñas atrapadas por el enamoramiento (vídeo "Viene un chico nuevo a clase, ¿quién será", en "Los Juguetes de Arantxa" -3,2M visualizaciones-), la moda, el maquillaje, el mundo de las princesas, representando el rol de amas de casa y cuidadoras del hogar y de los hijos (vídeos "Rutina de paseo con mi bebé Nenuco" y "24 horas siendo mamá, en "Los Juguetes de Arantxa” -656.171 visualizaciones-).

En cuanto a los estereotipos, se resaltan dos de manera llamativa. En el caso de "EthanGamer", se representa el estereotipo del niño con gafas aficionado a los videojuegos. De hecho, en la cabecera de sus vídeos se ve cómo nada más llegar del colegio se dispone a jugar. Por su parte, en el caso de "Los Juguetes de Arantxa", "Las Ratitas" y "La diversión de Martina", se evidencia el estereotipo de niñas cursis. Así, conviene puntualizar que se han detectado más estereotipos entre las youtubers niñas que en el caso de los niños.

La representación de roles y estereotipos puede influir en la definición y conformación de la identidad de quienes se encuentran en una fase crucial para su desarrollo, y es que los especialistas consideran que las concepciones sociales de lo que es un hombre y una mujer influyen a los pequeños desde los primeros meses de vida y creen que el impulso hacia lo femenino o lo masculino es consecuencia de lo que ven y oyen en televisión o en su contexto más cercano (en casa o en el colegio). Por ello, este estudio parte de la necesidad de analizar cómo se representan a los niños youtubers, personajes 
Inmaculada Sánchez, Labella Martín

Youtubers infantiles: los menores como recurso para generar nuevas tendencias publicitarias

de referencia para los usuarios. En este caso, se ha observado la representación de estereotipos según el género, lo cual resulta preocupante ya que los modos de ser y hacer que presentan perpetúan y normalizan, en un espacio de entretenimiento, determinadas actitudes consideradas propias del patriarcado, formando parte de su cotidianidad.

\section{Conclusiones}

Con todo lo expuesto, la investigación realizada confirma la H1 de manera parcial, ya que no todos los canales que conforman la muestra incluyen en todos sus vídeos marcas de productos y/o juguetes aptos para el público infantil. Por su parte, la H2 queda confirmada en su totalidad tras haber comprobado que todos los youtubers infantiles presentan modos de ser y hacer que se ajustan a los parámetros propios de los personajes creados para la ficción, lo que nos ha posibilitado su estudio según la dimensión iconográfica, psicológica y sociológica propuesta por los autores expertos en teoría narrativa para la construcción de personajes, mencionados anteriormente.

Así, se puede cerrar la investigación afirmando que YouTube se considera un espacio liderado por personajes que conforman una tribu en sí misma, la cual marca tendencia e influye en quienes ven dichas publicaciones. Si bien las tribus digitales se valen de las redes sociales para exponer y ofrecer sus contenidos, en el caso de la plataforma YouTube no hacen uso de ella para gestar redes y crear una comunidad, ya que la interacción, a través del diálogo con los usuarios, es nula. Se ha observado, además, cómo los youtubers infantiles han construido sus propios personajes, quedando sometidos al aumento de visitas y reproducciones. Al ir más allá, y sabiendo de la presencia de publicidad en estos espacios, los niños analizados -en su papel de youtubers- se han convertido, además, en personajes que incitan al consumo de determinadas marcas. Así, se entiende que estas los utilizan como vehículos para que la publicidad encaje en nuevos entornos digitales, suponiendo un nuevo modo de comunicación publicitaria.

Con todo ello, se concluye que los youtubers infantiles están presentes en YouTube valiéndose de este espacio como un trampolín para su reconocimiento, de ahí el ímpetu de este trabajo por demostrar que su intención principal es constituirse como personajes de referencia y que, para ello, recurren a la hegemonía que les proporciona esta plataforma. Este hecho ha propiciado que los youtubers infantiles hayan tenido que redefinirse, convirtiéndose en personajes debidamente construidos desde el punto de vista narrativo. Quienes guionan sus actuaciones, y más allá de la dimensión iconográfica, les han conferido una dimensión psicológica y sociológica sobre las que apoyar todas sus intervenciones, de manera que sus actuaciones quedan sujetas a una interpretación deliberadamente definida y alejada de la espontaneidad que los caracterizaba en sus orígenes; una actuación que en ocasiones se representa mediante estereotipos, lo que supone la transmisión de actitudes y comportamientos nocivos para 
Austral Comunicación

Volumen 9, número 2 (Diciembre de 2020): 249-274. ISSN 2313-9129

el público infantil. Por lo general, son preadolescentes dinámicos, creativos, activos y alegres que trabajan para continuar emitiendo contenido de interés para su público. Si bien en ocasiones los niños hacen de presentadores de productos, en otros casos crean historias en torno a estos. Esta configuración publicitaria se gesta previendo una mayor persuasión y, por tanto, aumento de ventas, ya que los prescriptores serán en este caso figuras altamente reconocidas. En cuanto a esto, se apunta un aspecto preocupante y es que no todas las marcas publicitarias presentes en los vídeos observados son aptas para el público infantil, lo cual nos hace reflexionar sobre la cosificación de estos niños por parte de las marcas comerciales.

Finalmente, y ante la preocupación por saber qué se cuenta y cómo se cuenta, se apunta la necesidad de más investigaciones centradas en analizar el contenido publicado desde un punto de vista narrativo.

\section{Referencias}

Asociación para la Investigación de Medios de Comunicación (AIMC) (2018). AIMC niñ@s 2018. España. Obtenido el 10 marzo de 2020 de https://www.aimc.es/ otros-estudios-trabajos/aimc-ninos/

Aran Ramspott, S., Fedele, M. y Tarragó, A. (2018). Funciones sociales de los Youtubers y su influencia en la pre-adolescencia. Revista Comunicar, 26(57), 71-80.

Aznar Díaz, I., Trujillo Torres, J. M., Romero Rodríguez, J.M. y Campos Sotos, M. N. (2019). Pixel-Bit. Revista de Medios y Educación, 56, 113-128.

Berzosa, M. (2017). Youtuber y otras especies: el fenómeno que ha cambiado la manera de entender los contenidos audiovisuales. Barcelona: Ariel.

Blades, M., Oates, C. y Li, S. (2013). Children's recognition of advertisements on television and on Web pages. Appetite, 62, 190-193.

Burgess, J. y Green, J. (2013). YouTube: Online video and Participatory Culture. Cambridge: Polity Press.

Casetti, F. y Di Chio, F. (2007). Cómo analizar un film. Barcelona: Paidós.

Chatman, S. (1990). Historia y discurso. La estructura narrativa en la novela y en el cine. Madrid: Taurus Humanidades.

Chau, C. (2010). Youtube as a participatory culture. New Directions for Youth Development, 128, 65-74.

Correa, T., Straubhaar, J., Chen, W. y Spence, J. (2015). Brokering new technologies: the role of children in their parents' usage of the internet. New Media \& Society, 17(4), 483-500.

eMarketerReport(2016).USKidsand Digital:GaugingHowDigital TheseDigitalNatives Really Are.Obtenido el 10 de marzo de 2020 de https:/www.emarketer.com/Report/ US-Kids-Digital-Gauging-How-Digital-These-Digital-Natives-Really/2001911. 
Inmaculada Sánchez, Labella Martín

Youtubers infantiles: los menores como recurso para generar nuevas tendencias publicitarias

Evans, N. J., Grubbs Hoy, M. y Carpenter Childers, C. (2019). Parenting "YouTube Natives": the impact of pre-roll advertising and text disclosures on parental responses to sponsored child influencer videos. Journal of Advertising, 47 1-21.

Feijoo, B. y Pavez, I. (2019). Contenido audiovisual con intención publicitaria en vídeos infantiles en YouTube: el caso de la serie Soy Luna. Communication \& Society, 32(1), 313-331.

Fernández Torres, M. J.y Chamizo Sánchez, R. (2020). Youtubers infantiles, los nuevos protagonistas de la comunicación comercial. Repositorio Institucional de la Universidad de Málaga.

Forbes (2017). Top Influencers: Kids. EE.UU. Obtenido el 10 de marzo de 2020 de https://www.forbes.com/top-influencers/2017/kids/\#53b906232643

Gallardo Camacho, J. (2013). Análisis del fenómeno YouTube en España: relación con los espectadores y con los generadores de contenidos tradicionales. Revista Luciérnaga, 5(9), 57-58.

Gallardo Camacho, J. y Jorge Alonso, A. (2010). La baja interacción del espectador de vídeos en Internet: caso YouTube España. Revista Latina de Comunicación Social, $65,421-434$.

Hargittai, E. y Hinnant, A. (2008). Digital inequality: differences in young adults' use of the Internet. Communication Research, 35(5), 602-621.

Hoffner, C. y Buchanan, M. (2005). Young Adults Wishful Identification with Television Characters: the role of perceived similarity and character attributes. Media Psychology, 7, 325-351.

Hudders, L. y Cauberghe, V. (2018). The Mediating Role of Advertising Literacy and the Moderating Influence of Parental Mediation on How Children of Different Ages React to Brand Placements. Journal of Consumer Behavior, 17, 197-210.

Jenkins, H. (2006). Convergence Culture. Where Old and New Media Collide. Nueva York: New York University.

Kunkel, D. y Castonguay, J. (2012). Children and advertising: content, comprehension and consequences. En Singer D. G. y Singer, J. L. (Eds.), Handbook of children and the media (pp. 395-418). Los Ángeles: Sage.

La Vanguardia (2019). Youtubers infantiles en el punto de mira. España. Obtenido el 9 de marzo de 2020 de https://www.lavanguardia.com/vida/20191014/47952595666/ youtuber-ninos-influencers-las-ratitas-mikeltube-juega-con-adri.html

León, L. (2018). Niños Youtubers y el proceso de creación de videos: evidencia de competencias transmedia en acción. Comunicación y Sociedad, 33, 115-137.

Livingstone, S. (2003). Children's use of the internet: reflections on the emerging research agenda. New Media \& Society, 5(2), 147-166.

Livingstone, S. (2009). Young people and the new media: processes of diffusion, 
Austral Comunicación

Volumen 9, número 2 (Diciembre de 2020): 249-274. ISSN 2313-9129

appropriation and use. (Translated into German). En Schorr, A. (Ed.), Grundlagen der Fugendmedienforschung-fünf Perspektiven (pp. 301-333). Alemania: VS Verlag für Sozialwissenschaften

Livingstone, S., Mascheroni, G. y Staksrud, E. (2015). Developing a Framework for Researching Children's Online Risks and Opportunities in Europe. Londres: EU Kids Online.

Lorente, C. (2017). Youtubers. Las nuevas estrellas mediáticas. Tiempo, 1788, 58-63.

Marketing4ecommerce (2020).20 niños youtubers que triunfan en España... yel mundo (2020). España. Obtenido el 9 de marzo de 2020 de https://marketing4ecommerce. net/10-ninos-Youtubers-que-triunfan-en-espana-el-mundo-2019/.

Marsh, J. (2016). Unboxing videos: co-construction of the chid as Cyberflâneur. Discourse: Studies in the Cultural Politics, 37(3), 369-380.

Martínez, C. y Olsson, T. (2019). Making sense of Youtubers: how Swedish children construct and negotiate the Youtuber Misslisibell as a girl celebrity. Journal of Children and Media, 13(1), 36-52.

Másquenegocio (2017). Las estadísticas de YouTube en 2017. España. Obtenido el 10 de marzo de 2020 de https://www.masquenegocio.com/2017/10/21/ estadisticas-youtube-2017/

Medrano, C. y Martínez de Morentín, J. I. (2012). Socialización y televisión: perfiles de adolescentes en un estudio transcultural. Revista Internacional Journal of Developmental and Educational Psychology, 1(1), 657-682.

Montes, M., García, A. y Menor, J. (2018). Los vídeos de los adolescentes en YouTube: características y vulnerabilidades digitales. Revista Comunicar, 24(54), 61-69.

O'Connor, C. (2017). Forbes Top Influencers: Meet the kids making millions reviewing toys, rapping and more. Obtenido el 10 de marzo de 2020 de https://www.forbes. com/sites/clareoconnor/2017/12/20/forbes-top-influencers-meet-the-kidsmaking-millions-reviewing-toys-rapping-and-more/\#75c20eeb4142.

Panic, K., Cauberghe, V. y Pelsmacker, P. (2013). Comparing TV ads and advergame targeting children: the impact of persuasion knowledge on behavioral responses. Journal of Advertising, 42(2-3), 264-273.

Pérez-Torres, V., Pastor-Ruiz, Y. y Abarrou-Ben-Boubaker, S. (2018). Los Youtubers y construcción de la identidad adolescente. Revista Comunicar, 24(55), 61-70.

Ramos, M. y Herrero, P. (2016). Unboxing and brands:Youtubers phenomenon through the case study of evantubehd. Prisma Social, Revista de Ciencias Sociales, 1 número especial, 90-120.

Raun, T. (2018). Capitalizing intimacy: New subcultural forms of micro-celebrity strategies and affective labour on YouTube. Convergence, 24(1), 99-113.

Rego, S. y Romero Rodríguez, L. M. (2016). Representación discursiva y lenguaje de 
los Youtubers españoles: estudio de caso de los gamers más populares. Index. comunicación, 6(1), 197-224.

Rodríguez, D. (2013). Memecracia. Los virales que nos gobiernan. Barcelona: Ediciones Gestión 2000.

Sabich, M. A. y Steinberg, L. (2017). Dircursividad Youtuber: afecto, narrativas y estrategias de socialización en comunidades de Internet. Revista Mediterránea de Comunicación, 8(2), 171-188.

Sáez, G. y Gallardo, J. (2017). La relación de los YouTubers con la publicidad y sus espectadores. El caso de YouTube España. Telos: Cuadernos de comunicación e innovación, 107, 47-57.

Sánchez,J.L. (2000). De la literatura al cine. Teoría y análisis de la adaptación. Barcelona: Paidós.

Sánchez Vera, M. M., Solano Fernández, I. M. y Recio Caride, S. (2019). El storytelling digital a través de videos en el contexto de la educación infantil. Píxel-Bit. Revista de Medios y Educación, 54, 165-184.

Segarra Saavedra, J.e Hidalgo Marí, T. (2018). Influencers, moda femenina e Instagram: el poder de prescripción en la era 2.0. Revista Mediterránea de Comunicación, 9(1), 313-325.

Suing, A., Salazar, G. y Ortiz, C. (2018). Are Latin American Youtubers Influential? En Antipode, T. y Rocha, A. (Eds.), Digital Science (pp. 341-348). Suiza: Springer.

Tur Viñes, V., Núñez Gómez, P. y González Ríos, M. J. (2018). Menores influyentes en YouTube. Un espacio para la responsabilidad. Revista Latina de Comunicación Social, 73, 1211-1230.

UNICEF (2017). Estado mundial de la infancia 2017: niños en un mundo digital. EE.UU. Obtenido el 10 de marzo de 2020 de https://www.unicef.es/sites/unicef.es/ files/comunicacion/estado-mundial-infancia-2017.pdf.

Westenberg, W. (2016). The influence of Youtubers on teenagers. (Tesis de Maestría). University of Twente. 